\title{
Outcomes from a Whole-Systems Ayurvedic Medicine and Yoga Therapy Treatment for Obesity Pilot Study
}

\author{
Jennifer Rioux, PhD, AD, AYT, IAYT, ${ }^{*}$ and Amy Howerter, $\mathrm{PhD}^{\dagger}$
}

\begin{abstract}
Objectives: To determine the feasibility and acceptability of an Ayurveda/Yoga intervention for weight loss, using dual-diagnosis inclusion criteria, dual-paradigm outcomes, and a semistandardized protocol with tailoring according to the Ayurvedic constitution/imbalance profile of each participant.

Design: Seventeen participants enrolled in a weekly intervention for 3 months. Outcome measurements were performed at baseline, postintervention, and 3 and 6 months follow-up.

Setting: The intervention was conducted through the University of Arizona, Department of Family and Community Medicine from April through December 2012.

Subjects: Participants included 2 men and 15 women recruited from the community of Tucson, AZ using flyers and hospital message boards. Seventeen enrolled and 12 participants provided complete follow-up data.

Intervention: Participants met with an Ayurvedic practitioner twice monthly (six times) and followed semistandardized dietary guidelines with individual tailoring to address relevant psychophysiological imbalances obstructing weight loss and a standardized protocol of therapeutic yoga classes three times weekly with recommended home practice of two to four additional sessions.

Outcome measures: Primary outcome was weight loss. Other biomedical outcomes included body mass index, body fat percentage, waist and hip circumference, waist to hip ratio, and blood pressure. Unique instruments were designed to collect data on outcomes associated with the Ayurvedic medical paradigm, including dietary changes by food qualities, mood/affect, relationships, and changes in Ayurvedic imbalance profiles.

Results: Participants lost an average of $3.5 \mathrm{~kg}$ during the 3 -month intervention. Weight loss at 3 and 6 months postintervention increased to an average of $5.6 \mathrm{~kg}$ and $5.9 \mathrm{~kg}$, respectively. Participants who lost $3 \%$ of their body weight during the 12 week intervention, lost on average an additional 3\% during the follow-up period. Psychosocial outcomes also improved. No additional services were provided to participants during the followup period.

Conclusions: A whole-systems Ayurvedic medicine and Yoga therapy approach provides a feasible promising noninvasive low-cost alternative to traditional weight loss interventions with potential added benefits associated with sustainable holistic lifestyle modification and positive psychosocial changes.
\end{abstract}

Keywords: Ayurveda, yoga, whole system, obesity, East Asian medicine, integrative medicine

\section{Introduction}

$\mathbf{O}$ BESITY IS AN EPIDEMIC IN THE U.S. population and has been designated as a research priority by the National Institutes of Health. ${ }^{1}$ The $\mathrm{CDC}$ reports that the prevalence of obesity was $39.8 \%$ and affected about 93.3 million of U.S. adults in 2015-2016, with an estimated annual medical cost of obesity in the United States at $\$ 147$ billion in 2008 U.S. dollars. ${ }^{2,3}$ Obesity is a primary causal factor in many diseases, including type 2 diabetes, hypertension, cardiovascular disease, dyslipidemia, stroke, and liver disease. ${ }^{4}$ Worldwide obesity has nearly tripled since 1975. In 2016, more than 1.9 billion adults, 18 years and older, were overweight. Of these $>650$ million were obese. Thirty-nine

Department of Family \& Community Medicine, University of Arizona, Tucson, AZ.

${ }^{*}$ Current affiliation: Integral Ayurveda and Yoga Therapy, Carrboro, NC.

${ }^{\dagger}$ Current affiliation: Quantitative Science, Clinical Outcomes Solutions, Tucson, AZ. 
percent of adults aged 18 years and over were overweight in 2016, and 13\% were obese. Most of the world's population lives in countries where overweight and obesity kill more people than underweight. ${ }^{5}$

According to a systematic review of 33 studies, the 4 highest quality studies calculated the per-person annual direct medical cost of obesity in 2008 as $\$ 1,723 .^{6}$ A 2014 study estimated that obese individuals may lose as much as 8 years from their life span due to comorbid conditions associated with obesity. ${ }^{7}$ Obesity and metabolic syndrome are preventable. ${ }^{5}$ Conventional care paradigms for obesity and metabolic syndrome treatment are expensive and may involve surgery and medications with side effects. ${ }^{8-10}$ Conventional medical approaches have demonstrated limited success in the treatment or prevention of obesity. ${ }^{11-13}$ Some trials have shown that even moderate weight loss of $5 \%-10 \%$ can significantly modify risk profiles for obesityassociated disease; thus, novel approaches to treat obesity are needed. $^{14}$

Inter- and multidisciplinary care have been shown to reduce weight and body mass index (BMI) ${ }^{15,16}$ with enhanced effects when combined with cognitive behavioral therapy. ${ }^{17}$ Obesity researchers now commonly acknowledge that tailored multimodal integrated interventions demonstrate the best outcomes. ${ }^{8-10}$ A 2005 study of numerous holistic healing modalities for which data were accessible showed that they appeared to be a good value, ${ }^{18}$ whereas a 2006 lifetime cost-use analysis from a societal perspective of outpatient weight loss strategies in overweight and obese U.S. women demonstrated that a multidisciplinary weight loss program consisting of diet, exercise, and behavior modification also provides good value for money. ${ }^{19}$

A 2018 analytic review of obesity management, diabetes prevention, and cardiovascular risk reduction concluded that, as a society, more is paid for disease management than disease prevention, and in failing to provide sufficient insurance coverage for weight management, the more costly management of a type 2 diabetes epidemic is funded. ${ }^{20}$

This whole-systems (WS) Ayurvedic medicine and Yoga therapy pilot intervention combined comprehensive lifestyle change; a first-time use of an Ayurvedically consistent cognitive-behavioral therapy approach (coined herein as Ayurvedic CBT) to addressing maladaptive emotions, behaviors, and cognitions associated with weight gain; and ritualized self-awareness practices with alternative diet and activity modification for overweight/obese adults. This intervention integrates the two complementary Vedic healing disciplines of Ayurveda and Yoga, which are linked through Samkhya philosophy and particular consideration of how disturbances of the mind inform disorders in the body and affect the doshas.

Particular portions of the classical text Charaka Samhi$t a^{21}$ relevant to the discipline of Ayurvedic Yoga therapy include (1) appropriate/inappropriate use of the senses and mental faculties, which are the essence of yogic practice and central to psychological and experiential aspects of disordered eating and sedentary lifestyle (Sa1 No. 118-132) and (2) the central role of yogic practice in pursuing selfunderstanding and escaping the cycle of attachment/ aversion, relevant to food cravings as well as addressing impulsive behavior and stagnation (rajas/tamas) (Sa1 No. 133-155). Thus, this application of whole-systems Ayur- veda and Yoga therapy (WSAY) has been applied in a manner consistent with traditional practice as described in Charaka Samhita ${ }^{21}$ and studies published in Indian medical journals that utilize asana to promote metabolic activity and affect doshic expression. ${ }^{22,23}$

This is the first published study to test the feasibility of a tailored WSAY weight loss intervention and presents promising preliminary results in terms of conventionally recognized outcomes for assessing obesity. This pilot study is also the first to collect standardized Ayurvedic outcomes in an academic medicine setting in the United States. Preliminary data analysis suggests that a whole-systems Ayurveda/Yoga approach to obesity offers an acceptable, noninvasive, and low-risk treatment option for obesity, through a semistandardized treatment protocol that is feasible to replicate, and potentially generalizable to broader populations.

A previous article on the study design and protocol discussed basic weight loss measures, adherence measures, participant demographics, and satisfaction with the intervention. $^{24}$ This article covers additional biomedical and Ayurvedic health outcomes and those related to process, context, and whole-systems features of the design, implementation, and analysis.

\section{Ayurvedic theory, obesity, and weight loss}

Ayurvedic medicine for treating obesity has not been rigorously studied in clinical trials, but other complex multimodal Ayurvedic treatments have been studied for efficacy and effectiveness in chronic conditions such as osteoarthritis, ${ }^{25}$ fibromyalgia, ${ }^{26}$ and hepatic cirrhosis. ${ }^{27}$ This intervention design offered an innovative and personalized obesity treatment approach focused on lifestyle changes, and diet and exercise modification, delivered according to the constitution/imbalance profile of the patient. ${ }^{28}$ This study utilized Ayurvedic Yoga Therapy, which integrates causal and therapeutic principles shared by these historically intertwined disciplines and is designated as a category of professional practice by the National Ayurvedic Medical Association. ${ }^{29}$

Yoga is inexpensive, noninvasive, and has been determined by two comprehensive reviews of clinical trials to be a safe effective intervention to reduce BMI and effective as primary or supplemental self-care for weight loss, weight maintenance, and prevention of obesity and metabolic syndrome. $^{30,31}$ Other studies of yoga for weight loss/metabolic syndrome/diabetes have found significant reductions in abdominal obesity, ${ }^{32}$ positive alterations in ghrelin axis, ${ }^{33}$ and a reduction in cardiometabolic risk factors. ${ }^{34}$ The specific mind-body approach of yoga therapy has also been found to spur psychosocial changes associated with durable weight loss, including "yoga culture" social support and reports that a yogic experience of weight loss was "easier" and "subjects felt more confident in their ability to maintain lasting weight loss.",35

The practice of Ayurvedic medicine entails the application of individualized, multimodal, and multitarget therapies and holds potential for the effective treatment of obesity due to its comprehensive approach. A 2015 commentary from the Lancet-Diabetes and Endocrinology provides clinical recommendations for obesity prevention and treatment, which 
specifically suggest "a multifaceted treatment strategy" and "individualized treatment." 36 An unrelated 10-week yoga program for weight loss, including "Ayurveda-inspired components" in a nonacademic setting, reported improvements in psychosocial outcomes and self-reported weight loss but was less comprehensive as an intervention, reported no objective measures, and included no Ayurvedic outcomes. $^{37}$

Ayurvedic theory focuses on the relationship between balanced and imbalanced expression of the five elementsair, space, fire, water, and earth — in the human body, mind, and spirit, as expressed in the doshas. The doshas are three bioenergetic systems that comprise pairs of elements and referred to as vata (air and space), pitta (fire and water), and kapha (water and earth). Ayurveda refers to obesity as Sthaulya (or Medoroga). Sthaulya is defined as a metabolic disorder stemming from lack of exercise, poor diet, stress, genetic predisposition, and ultimate increase in kapha dosha leading to excessive accumulation of adipose tissue, associated with accumulation of water and earth elements in the body-mind, poor digestive fire, and accumulation of toxins in the digestive tract. ${ }^{38-40}$

Overeating and a sedentary lifestyle contribute to the accumulation of earth and water elements in the body, as does excess sleep, steroid medications, and psychoemotional conditions such as depression and anxiety. Earth and water are associated with the heavy, dense, slow, and cold qualities and are antagonistic to strong metabolic fire, which is sharp, light, and hot, thus contributing to slow metabolism and obesity. ${ }^{41}$

\section{A multimodal tailored synergistic protocol}

The protocol utilized semistandardized treatment consistent with Ayurvedic medical theory, including isolated features of tailoring. This is consistent with real-world Ayurvedic clinical practice in which treatment implementation is responsive to patient feedback. Tailored implementation of the standardized intervention according to participant feedback is a hallmark of the Ayurvedic clinical approach, and is a consistent feature of holistic/integrative health care delivery. ${ }^{42}$ Ayurveda and Yoga are complex interventions, including multifactorial components, which mutually enhance one another to achieve synergistic therapeutic effects. As healing modalities, Ayurveda and Yoga focus on the cumulative effects of multiple agents-diet, lifestyle, self-awareness, postures, breathing techniques, and meditation-acting simultaneously on the individual as a biopsychosocial network. $^{43}$

Ayurvedic and Yogic treatments are consistent with systemsbased models of obesity causality that inform optimal treatment frameworks. ${ }^{15-17}$ Multiple causal factors mutually enhance and reinforce one another in a synergistic manner in the overweight/obese individual. Ayurveda and Yoga provide exercise, stress reduction, nervous system balance, shifting of metabolic function, and anti-inflammatory effects to address many of the root causes associated with obesity. ${ }^{44}$ The match between a systems approach to addressing the multiple causal factors of obesity and the systems approach to treatment inherent within Ayurvedic medicine and Yoga therapy may provide optimal therapeutic effects (Fig. 1).

\section{Materials and Methods}

\section{Study design and sample}

This study was a prospective single-arm pre/post design with longitudinal follow-up at 3 and 6 months after the intervention. The study design incorporated a protocolized approach to diet modification and yoga therapy, as appropriate for the predetermined Ayurvedic constitution of primary or secondary kapha dosha and a concomitant imbalance of elevated kapha dosha. Study participants were subjected to dual-diagnosis assessment that included a BMI of 25-45, indicating overweight through morbid obesity. The intervention

\section{Ayurvedic Treatment and Clinical Outcomes}

Dynamic Assessment

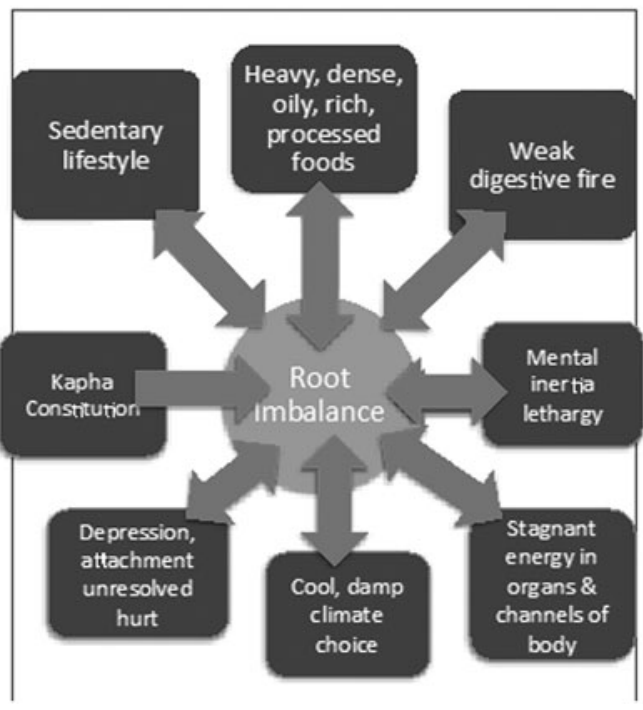

Synergistic Treatment

FIG. 1. Systems approach to Ayurvedic treatment and clinical outcomes.

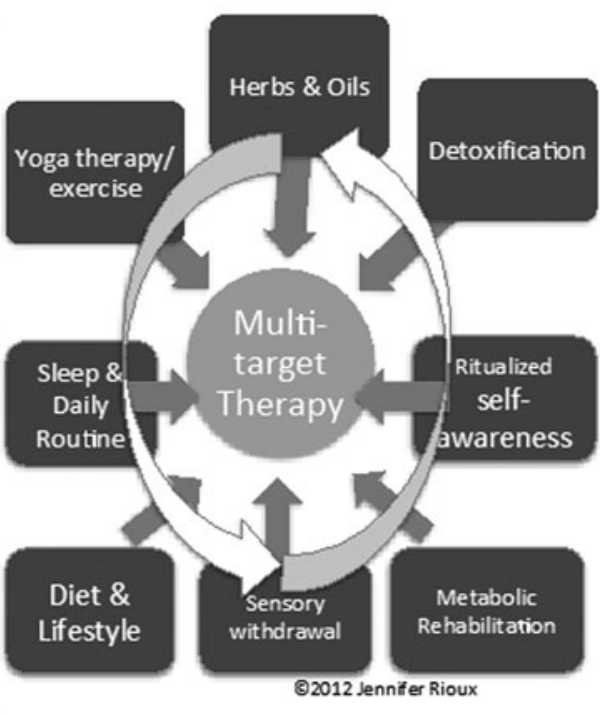


was semistandardized, in that diet and yoga protocols for reducing kapha were further tailored to the needs of the individual based on variance in constitutional type, particular food habits/needs, and physical functionality as related to yoga.

Recruitment for the study included posting notices for a "holistic weight loss program" on university hospital message boards and through flyers in the community. Participants were overweight/obese adult community members, naive to Ayurveda and Yoga, and were paid no fees for the intervention. If interested, participants were told they would participate in a 3-month intervention combining Ayurvedic diet, lifestyle modification, and yoga therapy, and would have follow-up assessments after the intervention was complete. This pilot feasibility study was approved by the University of Arizona Institutional Review Board. All study participants provided consent before participation.

\section{Inclusion/exclusion criteria}

Study participants met both biomedical and Ayurvedic inclusion and exclusion criteria. Inclusion criteria consisted of a biomedical diagnosis of obesity through BMI between 25 and 45 and a traditional Ayurvedic pulse analysis demonstrating either primary or secondary prominence of kapha dosha in the constitution and aggravated kapha dosha (increased water and earth elements) as primary in the imbalance. Ayurvedic pulse analysis is a traditional diagnostic method by which a trained clinician assesses pulse characteristicsmovement, rate, rhythm, force, volume, tension, temperature, consistency - under the index, middle, and ring fingers, associating these with doshic qualties, such as cold/hot, light/ heavy, and mobile/slow. ${ }^{45}$

To ensure consistency, constitution/imbalance profiles were assessed through pulse by 1 Ayurvedic doctor with 12 years experience in pulse assessment. This pilot study represents the first time that Ayurvedic pulse diagnosis was collected as an outcome in a clinical trial. Pulse assessment subsequently appeared in an unrelated pilot study of Ayurveda for coronary heart disease. ${ }^{46}$ Exclusion criteria included insulindependent diabetes; arthritis or osteoporosis compromising functionality; current cardiovascular disease; issues with balance/equilibrium or mobility; yoga practice within the past 2 years; other type of current weight loss treatment; and age $>70$ years.

\section{Intervention}

The intervention was designed as a comprehensive diet, activity, and lifestyle modification program based on principles of Ayurvedic medicine and Ayurvedic Yoga therapy. This intervention combined several features, which have subsequently been identified as essential to weight loss interventions: (1) personalized nutrition, (2) a problemsolving and motivational approach to lifestyle adherence, and (3) targeted strategies for reducing food cravings. Tailored nutrition prescription, defined as "comprehensive and dynamic nutritional recommendations based on shifting, interacting parameters in a person's internal and external environment" (47:93), closely matches the Ayurvedic approach to personalized dietary modification based on constitution/imbalance profiles.

Precision nutrition has been shown through a systematic review to be promising for the prevention and management of metabolic disorders. ${ }^{47}$ This WSAY intervention combined Ayurvedic CBT as individualized solution-focused coaching with motivational social support delivered in group yoga classes. A subsequent pilot study of a coaching approach to weight loss delivered through group visits in primary care led to significant weight loss and was found to be efficient and cost effective. ${ }^{48}$ Ayurvedic strategies for identifying and addressing food cravings and aversions, according to the science of food qualities, were an important aspect of the intervention, presented graphically in Figure 2. Cravings and aversions have recently been described as "food cue reactivity" by neurobiologists citing their critical role in obesity. ${ }^{49}$

Ayurvedic CBT involved significant ritualized selfawareness and self-monitoring of lifestyle behaviors. Combining practitioner-led patient education and individualized coaching reinforced group social support provided in yoga classes. Intervention components were the semistandardized Ayurvedic diet approach, three 75 min group yoga classes per week supplemented by home practice, and twice monthly visits over 12 weeks with an Ayurvedic Doctor (AD). The AD utilized Ayurvedic CBT and traditional Ayurvedic diagnostic methods, including assessment of doshic imbalances through pulse and tongue analysis to chart physiological and psychosocial changes in response to treatment over time.

The Ayurvedic diet reduced or eliminated foods that aggravate earth and water elements and increased the use of spices and freshly prepared foods to stoke metabolic fire and remove toxins from the digestive tract (Appendix A). The Ayurvedic doctor/Ayurvedic Yoga therapist with 12 years' experience provided patient care and some yoga instruction with support from another yoga professional trained in the protocol. Both researchers were blinded to all outcomes until follow-up data collection was complete.

Tailored advice during Ayurvedic consultations included therapeutic recommendations regarding sleep, food cravings, daily routine, sensory input, relationships, and selfawareness informed by participant feedback and discussion of barriers to adherence protocol. The implementation, sequencing, and specific instructions for Ayurvedic Yoga therapy were delivered in a stepped approach as yoganaïve participants increased their endurance. Yoga instruction was also tailored to participant functionality, using well-established props and modifications to make postures accessible (Appendix B).

Yoga therapy treats obesity through postures, breathing techniques, and meditation that improve muscle tone, reduce fat, regulate the nervous system and psychoemotional states, and detoxify tissues/improve metabolism. ${ }^{30-34}$ Ayurvedic Yoga further addresses obesity by counteracting the slow, static, cold, heavy, and dense qualities. ${ }^{22,23}$ The intervention was designed to change eating and activity patterns and to improve self-efficacy, quality of life/well-being, vitality, stress management, self-awareness around food choices, and barriers to weight loss. A comprehensive multimodal Ayurvedic approach, including CBT and tailoring treatment to the physiology and psychoemotional profile of the individual, potentially increases therapeutic impact and maximizes the accessibility and sustainability of change for the participant. $^{8-10,15-17}$ 

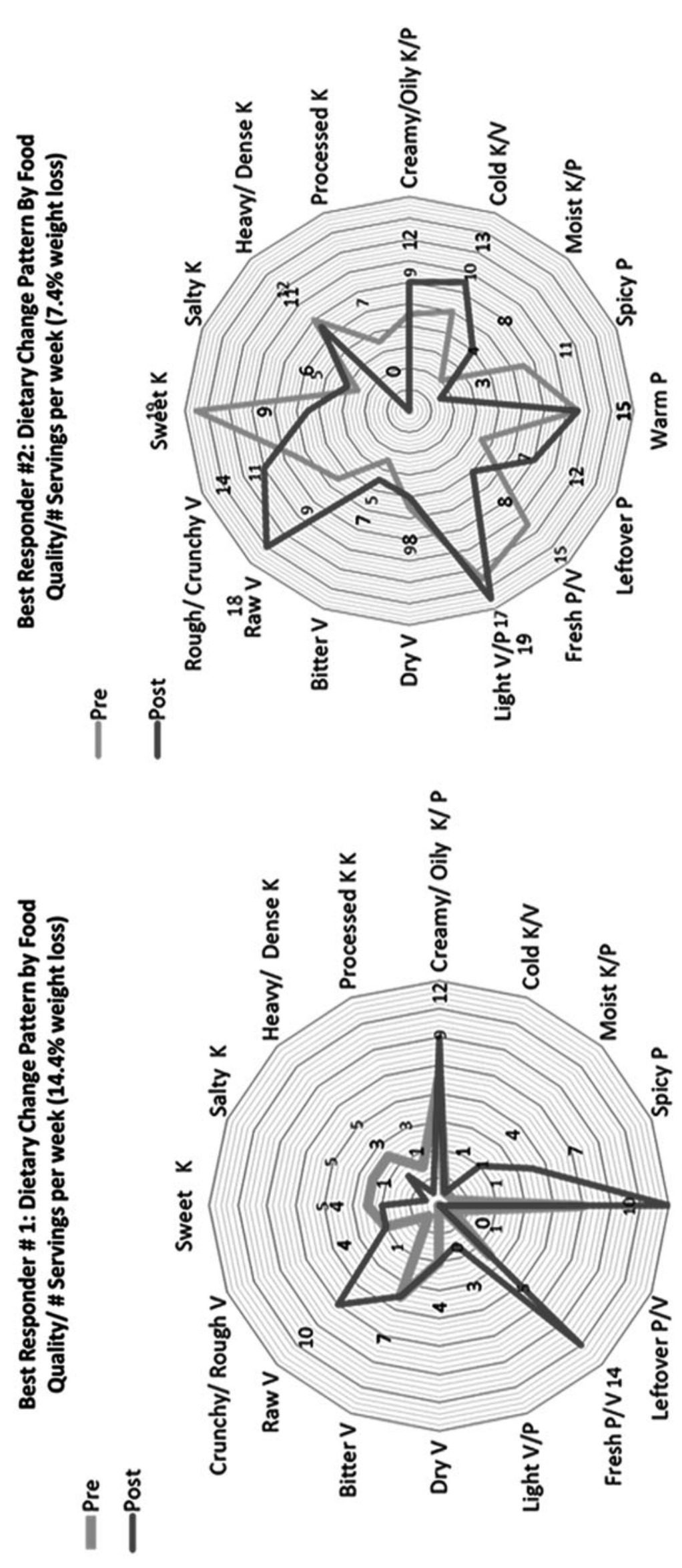

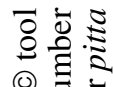

(ㅇ) 링

을

능

웜

트

영 용

吾焉

至壱

$4 \otimes$

$\infty_{00}$ 范

矛要

:⿹

응 흐

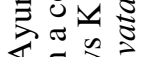

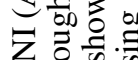

元

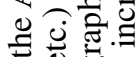

.0

๑

造语:

¿े పี

的本 을

$\Xi 3: \frac{0}{3}$

它气

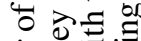

站

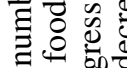

㭉导

능휴

氜

흘

후워

空志氞

응 흥 흥 융

a $\quad$ 。

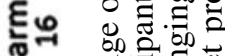

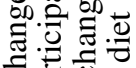

入气 ญ

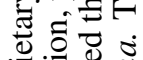

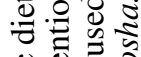

.0

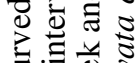

胥里

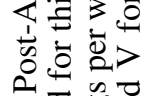

ค.

ن. 


\section{Data collection procedures}

Standardized Ayurvedic outcome instruments were developed to collect data on diet, use of senses and relationships to reflect model validity, and explore connections between physiological and psychosocial outcomes of Ayurvedic treatment. Other Ayurvedic outcomes such as pulse analysis, hydration, strength of appetite, and elimination patterns were practitioner collected and did not require unique instruments, but were critical in understanding paradigmspecific causality. ${ }^{42,43}$ Ritualized self-awareness practices of the WSAY intervention were integrated into data collection processes. Participants used the diet data collection tools to enhance their experience and understanding of food qualities and how they contribute to weight gain/loss, supporting increased motivation and self-efficacy. (Fig. 2).

Recording frequency and duration of yogic practices such as breath work, meditation, and postural practice supported self-regulation. Participant-led data collection increased five traits that a 2015 review found to predict long-term weight loss success: (1) autonomous motivation, (2) self-efficacy, and (3) self-regulation for weight and physical activity outcomes, and (4) positive body image and (5) flexible eating restraint for sustainable effects on weight control (50:84). Ayurveda-consistent self-monitoring and data collection procedures were consistent with noted psychological mediators of successful outcomes in obesity-related lifestyle-change interventions. ${ }^{50}$

Self-report instruments were designed to capture data in five lifestyle-related areas identified by Ayurvedic medicine as potential contributors or impediments to weight loss: (1) dietary intake based on food qualities, including cravings and aversions (Fig. 2); (2) changes in mood associated with kapha aggravation; (3) media usage and excess sensory stimuli; (4) frequency, content, and intensity of yoga, breathing, and meditation; and (5) changes in relationship quality and interaction. ${ }^{22,23,38-40}$ Data were also collected through visual analog scales (VAS) with a range of 1-100 on appetite, energy, and self-awareness to represent nonspecific effects of the intervention or overall benefit. ${ }^{51}$ Data were collected through Ayurvedic practitioner assessment, as well as patient self-report, across many time points.

This multireporter multitime-point strategy of data collection allows for a layered description of health outcomes, which contain both objective and subjective measures, as well as process outcomes and contextualizing information. This has been referred to as the evidence mosaic, ${ }^{52}$ characterized by overlapping analyses of data sets and sources. The evidence mosaic also resonates with the concept of topographical data collection intentionally used in this study design, ${ }^{24}$ which includes multiple overlapping sources of data that form a composite picture of the overall phenomena and do not dismiss or exclude data that are emergent within the research process, rather than predetermined.

These data combine to encompass both patient and provider priorities and provide a rich frame of reference for understanding aspects of the health care experience, the treatment paradigm, or environmental variables that arise as emergent properties of the whole system. One example of an emergent property resulting from comprehensive Ayurvedic diet and lifestyle change is that family members notice increased vitality or weight loss in the study participant, thus developing an affinity for the new habits of their family member or an interest in yoga. This change in perspective increases social support for change, thus creating an impetus for greater adherence to the protocol, begetting additional improvements in weight loss and vitality.

Provider-reported outcomes included changes in body weight, BMI, body fat percentage, fat/lean mass, waist/ hip circumference and ratio, and blood pressure. Patientreported outcome measures included diet and exercise selfefficacy scales adapted from Bandura's work on health promotion through social cognitive means ${ }^{53}$ and modified to include Ayurveda-consistent categories of analysis associated with kapha dosha; the Perceived Stress Scale (PSS-10) 10 items rated from 0 never to 4 very often ${ }^{54}$; Pittsburgh Sleep Quality Index (PSQI); $100 \mathrm{~mm}$ line VAS of energy, appetite, stress, quality of life, well-being, and program satisfaction at all time points. ${ }^{51}$

Uniquely designed instruments were used to collect data on diet by food qualities (Fig. 2), sensory input (e.g., frequency, duration and intensity of TV or music), changes in kapha mood/emotions (e.g., confused, stuck, joyous, content), primary relationships (\% of time experienced as positive vs. negative), and sleep patterns (No. of hours sleep per night/No. of sleep interruptions). Adherence to thrice weekly yoga classes, home yoga practice, and bimonthly consultations with an Ayurvedic medicine practitioner was recorded by study staff. Anthropometric, psychosocial, and Ayurvedic outcomes were analyzed to highlight relationships between variables, including therapeutic context. ${ }^{24}$

\section{Data analysis}

In this feasibility study, the dual-diagnosis design provided a diagnostic rubric that informed the development of the standardized intervention, whereas the psychosocial context of each patient and their response to treatment informed the individualization of the intervention as it proceeded. The patient population represented a cohesive whole (through similarity of constitutional/imbalance profiles), whereas tailored treatment features provided the opportunity to maximize patient response to, and compliance with, the intervention. Intensive multilayered (e.g., topographic/mosaic) collection of clinical outcomes provided insight into individualized features of the treatment regimen, whereas the density of data on any given feature of the intervention, or treatment response, allowed for optimal interpretation of outcomes in relation to a predetermined causal model.

Outcomes were analyzed to explore synergy of therapeutic components and patient priorities shaped how the flexible features of the intervention were implemented. For instance, as participants changed their dietary habits, their elimination improved; better elimination led to less toxicity in the body; less toxicity in the body informed better moods; better moods encouraged optimal decision making around food; optimal decision making around food increased the potential for weight loss; weight loss increased energy and activity levels, thereby improving yoga attendance and intensity; and so on. Figure 3 shows the principle of mutual causality, an iconic feature of whole systems in which change is bidirectional, with any therapeutic improvement having the potential to positively impact another salutogenic process. 


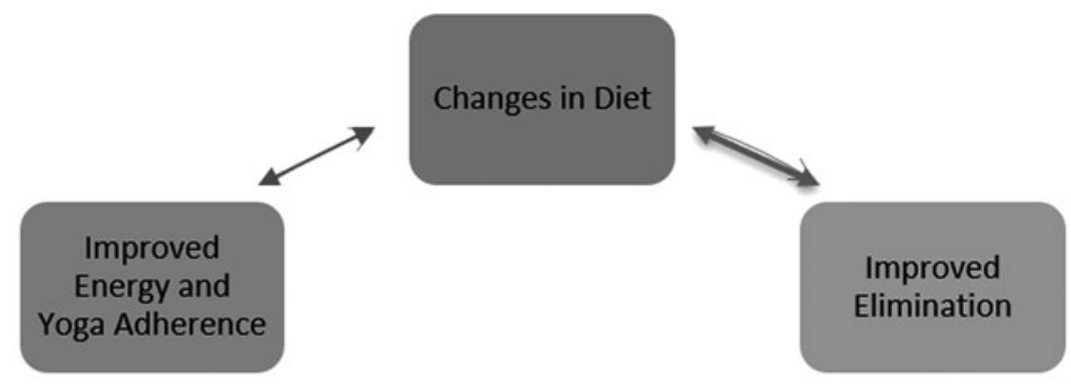

FIG. 3. Multimodal Ayurvedic approach to obesity. Demonstrates the mutual causality and bidirectionality that is an iconic feature of whole systems of medicine, necessitating certain research design and analysis features to accurately represent the therapeutic process.

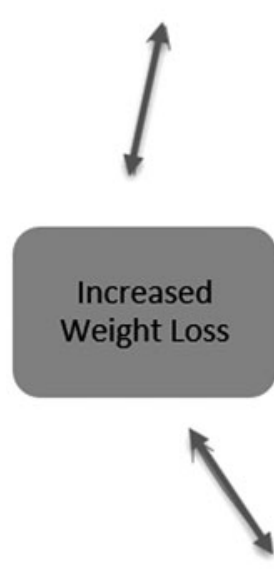

Optimal

Decision Making

Better Moods

As this is a pilot study with 17 participants enrolled, within-person difference scores were calculated for each time point and descriptive statistics are provided, but an a priori decision was made by the research team to not perform statistical comparisons as this is a pilot study with a small sample size. Descriptive statistics included mean, standard deviations, frequency, percentage, and change scores. Analysis of the follow-up period was designed to evaluate the durability of the effect, that is, maintenance/ continuation of weight loss and lifestyle changes. Exploratory and visualization analyses were performed to measure synergy between outcomes and comparisons of Ayurvedic concepts that do not lend themselves to traditional computational analysis.

WS concepts, such as tipping points of behavioral change, or identification of thresholds for intervention effectiveness, are explored here through visual analysis using the relationship between adherence, lifestyle changes, and yoga practice. Ayurvedic lifestyle outcomes such as profiles of constitution and imbalance are graphically displayed. All computations were performed using Stata 12 and graphics were created in Stata 12 and Microsoft Excel.

\section{Results/Outcomes}

Seventeen participants enrolled in the study. Participants were 41.3 years of age on average with 2 men and 15 women. Overall, participants lost an average of $3.5 \mathrm{~kg}$. during the duration of the intervention (Table 1). Weight loss had increased to an average of $5.6 \mathrm{~kg}$ and $5.9 \mathrm{~kg}$. at 6 and 9 months, respectively, with no additional services provided. Mean weight loss at 6 months postintervention was $6 \%$. It has been noted by biomedical clinicians that weight loss as minimal as $5 \%-10 \%$ of initial weight is sufficient for clinically meaningful reductions in weight-related biomarkers. ${ }^{8,13}$ Aggregate blood pressure (practitioner-reported) was $123 / 81$ at baseline, $114 / 74$ postintervention, $113 / 74$ at 6 months, and $118 / 81$ at 9 months.

On average, self-efficacy around dietary change and exercise improved in all categories from baseline to 3 months with continued improvement at 6 months and minimal decline at 9 months, indicating a need for additional support during the follow-up period. Changes in perceived stress improved from baseline to 3 months and ability to maintain positive outlook was sustained at 6 and 9 months. Participant self-report of stress indicated reduced stress levels at every time point after enrollment. Continued weight loss and maintenance of improved psychosocial outcomes were sustained at 9 months, indicating durability of health outcomes over time. Participants reported increased energy, well-being, and quality of life, at 3 and 6 months, with measures above baseline values at 9 months.

\section{Intervention adherence and weight loss}

To explore the relationship between adherence to the program and weight loss, each participant's weight was triangulated on a graph (Fig. 4) with yoga program adherence ( $x$ axis) and dietary recommendation adherence $(y$ 
Table 1. Anthropometric and Conventional Outcomes

\begin{tabular}{|c|c|c|c|c|}
\hline & $\begin{array}{c}\text { Baseline } \\
\text { Mean (SD) }\end{array}$ & $\begin{array}{c}3 \text { Month } \\
\text { Mean (SD) }\end{array}$ & $\begin{array}{c}6 \text { Month } \\
\text { Mean }(S D)\end{array}$ & $\begin{array}{l}9 \text { Month } \\
\text { Mean (SD) }\end{array}$ \\
\hline \multicolumn{5}{|l|}{ Anthropometric } \\
\hline Weight (lbs.) & $205.1(42.0)$ & $197.4(39.5)$ & $192.0(40.3)$ & $184.9(69.6)$ \\
\hline Weight change (lbs.) & & $-8.3(10.0)$ & $-13.1(13.8)$ & $-13.0(17.9)$ \\
\hline Weight change $(\%)$ & & $-3.8 \%(0.04)$ & $-5.6 \%(0.07)$ & $-6.2 \%(0.09)$ \\
\hline BMI & $33.2(5.2)$ & $31.9(4.8)$ & $31.2(5.6)$ & $31.3(5.4)$ \\
\hline Body fat $\%$ & $43.2(5.6)$ & $39.4(4.7)$ & $39.7(6.7)$ & $39.4(6.1)$ \\
\hline Waist circumference $(\mathrm{cm})$ & $112.4(15.1)$ & $107.0(14.3)$ & $106.5(17.2)$ & $103.2(15.8)$ \\
\hline Waist circumference change $(\mathrm{cm})$ & & $-5.4(7.1)$ & $-5.8(8.4)$ & $-9.0(10.5)$ \\
\hline Hip circumference $(\mathrm{cm})$ & $121.4(13.8)$ & $117.7(10.5)$ & $116.0(12.1)$ & $115.7(12.2)$ \\
\hline Hip circumference change $(\mathrm{cm})$ & & $-3.7(5.9)$ & $-5.4(6.2)$ & $-4.7(6.6)$ \\
\hline Waist to hip ratio & $0.91(0.05)$ & $0.89(0.06)$ & $0.91(0.08)$ & $0.89(0.08)$ \\
\hline WHR change & & $-0.18(0.03)$ & $0.00(0.04)$ & $-0.02(0.05)$ \\
\hline \multirow{2}{*}{\multicolumn{5}{|c|}{$\begin{array}{l}\text { *11 people total at } 6 \text { months: } 1 \text { dropout (surgery); } 10 \text { people at } 9 \text { months: }+1 \text { dropout (relocation) } \\
\text { Blood pressure }\end{array}$}} \\
\hline & & & \multicolumn{2}{|c|}{ Blood pressure } \\
\hline Blood pressure—systolic & $123(19)$ & $114(13)$ & $113(15)$ & $118(12)$ \\
\hline Blood pressure—diastolic & $81(9)$ & $76(10)$ & $74(6)$ & $81(9)$ \\
\hline \multicolumn{5}{|l|}{ Psychosocial/stress } \\
\hline Well-being & $66.3(12.9)$ & $78.8(7.8)$ & $77.3(17.2)$ & $73.1(12.8)$ \\
\hline Quality of life & $75.2(12.8)$ & $82.1(6.2)$ & $85.3(10.5)$ & $76.1(11.5)$ \\
\hline Energy & $67.9(13.6)$ & $74.5(8.4)$ & $68.2(21.2)$ & $63.6(21.1)$ \\
\hline \multicolumn{5}{|l|}{ Self-efficacy } \\
\hline \multicolumn{5}{|l|}{ Eating } \\
\hline Relationship with others/self & $60.8(12.9)$ & $66.5(15.5)$ & $67.5(15.3)$ & $58.8(23.7)$ \\
\hline Adapt to routine/circumstance & $68.8(14.3)$ & $74.4(10.4)$ & $75.4(12.3)$ & $69.3(13.6)$ \\
\hline \multirow{2}{*}{\multicolumn{5}{|c|}{ Exercise }} \\
\hline & & & & \\
\hline Relationship with others/self & $65.8(20.2)$ & $67.2(12.7)$ & $69.1(20.6)$ & $59.8(18.9)$ \\
\hline Adapt to routine/circumstance & $64.9(16.4)$ & $68.5(12.2)$ & $65.6(18.4)$ & $59.9(22.8)$ \\
\hline Internal/emotional regulation & $56.3(16.9)$ & $66.5(13.4)$ & $60.9(15.9)$ & $52.9(18.8)$ \\
\hline \multicolumn{5}{|l|}{ Secondary outcomes } \\
\hline Water intake (oz. per day) & $42.3(27.6)$ & $62.8(26.2)$ & $51.8(27.7)$ & $52(30.2)$ \\
\hline Appetite (VAS 0-100) & $75.8(14.1)$ & $69.2(11.8)$ & $73.8(10.9)$ & $75.8(12.7)$ \\
\hline Elimination (BM/× per day) & $1.3(0.77)$ & $2.0(1.2)$ & $1.8(1.2)$ & $1.5(0.52)$ \\
\hline
\end{tabular}

*denotes change in $N$ during follow up.

$\mathrm{BM}$, bowel movement; BMI, body mass index; VAS, visual analog scales; WHR, wait-to-hip ratio.

axis). Each participant's weight change was displayed for the 3,6, and 9 month follow-up periods. Those with negative change scores indicate weight loss, and those with positive scores indicate weight gain. Visual analysis of the pattern clustering indicates a positive relationship with a minimum of $55 \%$ adherence to both yoga and dietary components and weight loss.

\section{Ayurvedic constitution and imbalance data}

There are a number of ways in which doshic expression through the constitution and imbalance profiles of the patient can be expressed. The Ayurvedic doctor/researcher in this intervention was trained to characterize the expression of the doshas in the patient constitution as a ratio of $3: 2: 1$, with the most prominent dosha as 3, the second most prominent dosha as 2, and the least prominent dosha as $1 .^{41}$ Doshic expression in the patient imbalance through this method is characterized as quantitatively above the baseline measures of the patient's constitution, and is measured by assessing qualities of the pulse under index, middle, and ring fingers including movement, rate, rhythm, force, volume, tension, temperature, and consistency, as noted above. All study participant pulse analysis was conducted by one Ayurvedic doctor to maintain consistency and the doctor was blinded to changes in patient pulse analysis until data collection was complete.

Analysis of the aggregate constitutional pulse in Figure 5 shows that the study sample was predominantly pitta with secondary kapha and tertiary vata. Individuals with any constitution may develop disorders of any doshic type; however, it is more common for individuals who have primary or secondary kapha to develop a kapha imbalance. An individual's original constitution represents a state of balance or health for that person. The numbers associated with the constitutional baseline hereunder (column 1) represent therapeutic targets of doshic balance or health, expressed as numbers.

Analysis of the aggregate imbalance (column 2) shows that in the study participant group as a whole, there was evidence of aggravation in all three doshas with kapha (earth and water) being most elevated $(+1.08)$, vata second 


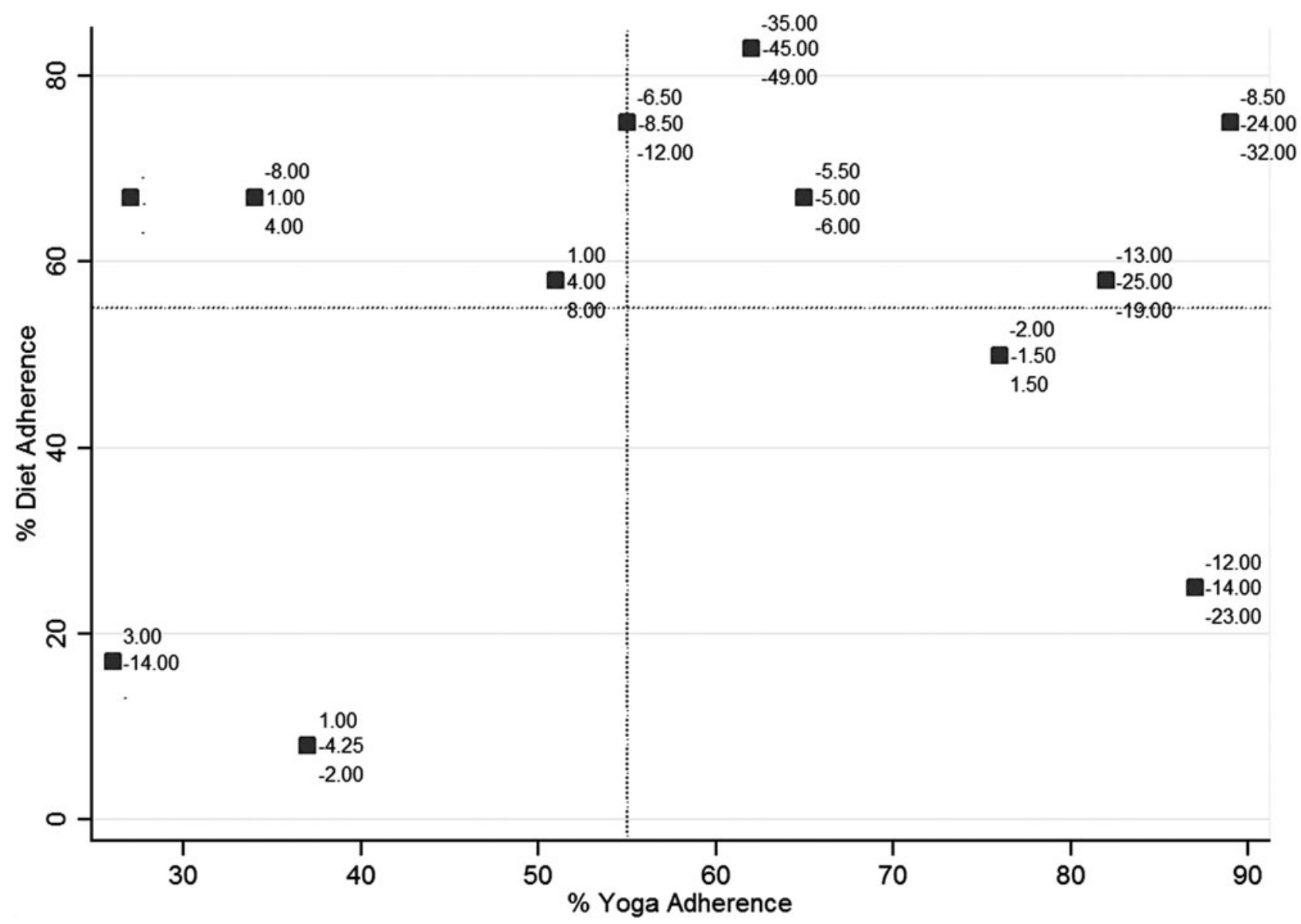

FIG. 4. Weight loss as a function of diet and yoga adherence. Weight loss (lbs.) is displayed for 3 months (top), 6 months (middle), and 9 months (bottom) for each participant. Participants with $>55 \%$ adherence in both diet and yoga demonstrate the greatest levels of weight lost and maintained after the intervention. Numbers associated with each box show weight loss in pounds at the three data collection time points. This graph shows the tipping point of $3 \%$ loss of baseline body weight $(N=11)$. (One participant's data excluded due to multiple surgeries during intervention.)

FIG. 5. Composite constitution/imbalance data.

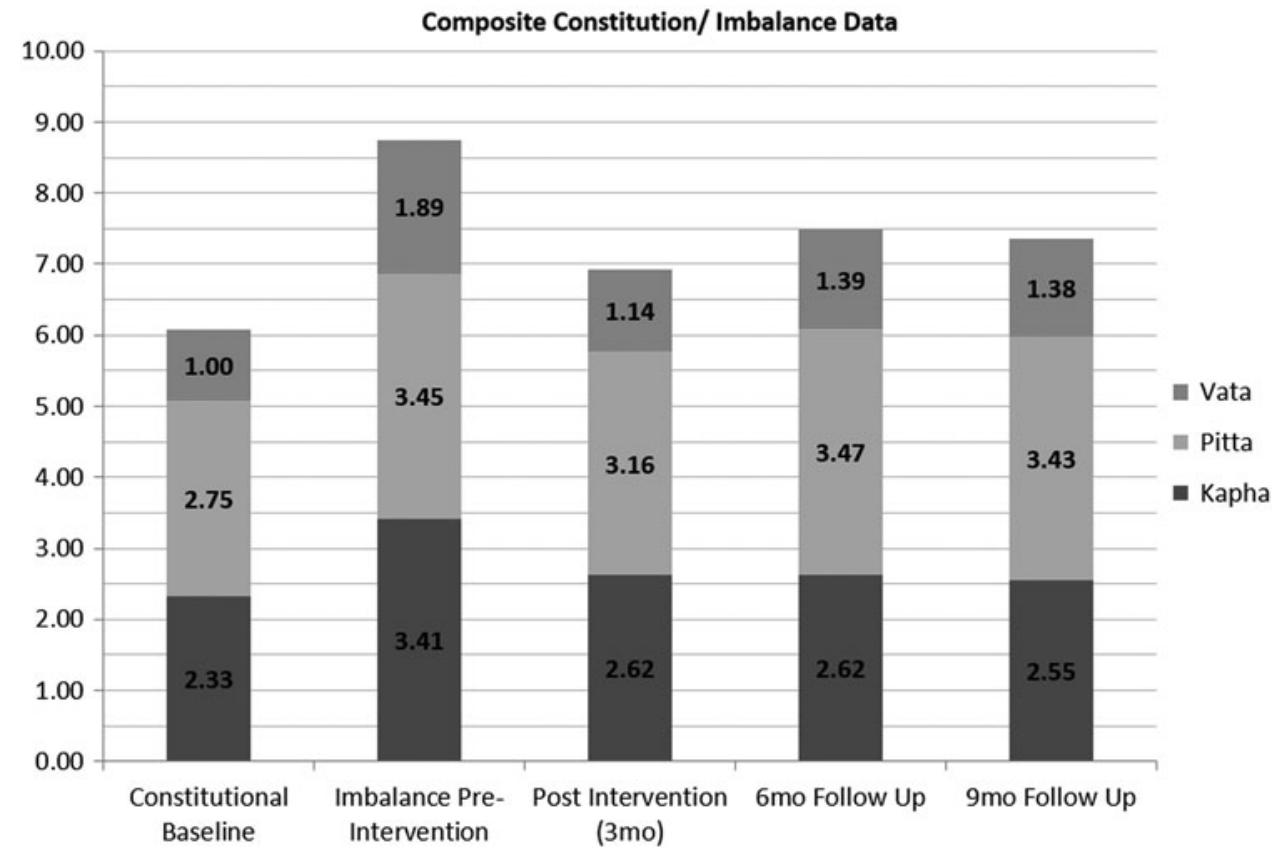


Table 2. Ayurvedic Outcomes

\begin{tabular}{|c|c|c|c|c|}
\hline \multirow{2}{*}{ Doshic change (pulse analysis) } & Vata & \multicolumn{2}{|r|}{ Pitta } & Kapha \\
\hline & 1 & & 2.75 & 2.33 \\
\hline Aggregate imbalance baseline & 1.89 & & 3.45 & 3.41 \\
\hline Aggregate imbalance 3 months & 1.14 & & 3.16 & 2.62 \\
\hline Aggregate imbalance 6 months & 1.39 & & 3.47 & 2.62 \\
\hline Aggregate imbalance 9 months & 1.37 & & 3.42 & 2.55 \\
\hline Sleep quantity/awakenings & & Pre & & Post \\
\hline Average No. of hours slept per night & & $7.06(0.69)$ & & $7.58(0.29)$ \\
\hline No. of subjects waking in night $N=12$ & & $12(0.63)$ & & $6(0.58)$ \\
\hline \multicolumn{5}{|l|}{ Ayurvedic mood/affect scale } \\
\hline \multicolumn{5}{|l|}{ Imbalanced kapha emotions } \\
\hline Frequency of reporting $(N=12)$ & & $5.75(1.71)$ & & $2.75(0.96)$ \\
\hline Magnitude $(0-20$ scale $)$ & & $8.81(0.98)$ & & $7.38(3.33)$ \\
\hline \multicolumn{5}{|l|}{ Imbalanced kapha cognitive dysfunction } \\
\hline Frequency of reporting $(N=12)$ & & $7.66(1.16)$ & & $4.33(1.53)$ \\
\hline Magnitude $(0-20$ scale $)$ & & $8.82(0.66)$ & & $9.16(1.35)$ \\
\hline \multicolumn{5}{|l|}{ Balanced kapha internal/emotional regulation } \\
\hline Frequency of reporting $(N=12)$ & & $8(1.83)$ & & $7.25(2.5)$ \\
\hline Magnitude $(0-20$ scale $)$ & & $11.43(2.57)$ & & $12.8(1.93)$ \\
\hline \multicolumn{5}{|l|}{ Balanced kapha relationships with others/self } \\
\hline Frequency of reporting $(N=12)$ & & $5.5(1.0)$ & & $5.25(1.71)$ \\
\hline Magnitude (0-20 scale) & & $7.8(2.66)$ & & $13.5(1.24)$ \\
\hline $\begin{array}{l}\text { Quality of relationships: changes } \\
\text { pre to post }\end{array}$ & $\begin{array}{l}\text { Positive \% } \\
\text { per week }\end{array}$ & $\begin{array}{l}\text { Negative } \% \\
\text { per week }\end{array}$ & $\begin{array}{c}\text { Intensity, } \\
\%\end{array}$ & $\begin{array}{l}\text { Nonintentional } \\
\text { interaction, \% }\end{array}$ \\
\hline Family/personal & +7.07 & -9.96 & +11 & -30 \\
\hline Work/school & +9.9 & -3.38 & -19.5 & -9 \\
\hline Health/food & +3.21 & -28.23 & -22.5 & -6 \\
\hline Self & +6.77 & -11.50 & -5 & -13 \\
\hline
\end{tabular}

most elevated (+0.89), and pitta least elevated $(+0.7)$. This is consistent with the understanding of obesity as a kapha disorder, with underlying stress and nervous system aggravation (vata) that disrupts optimal metabolic activity (pitta). Columns 3, 4 and 5 represent an assessment of aggregate imbalance at 3 (postintervention), 6, and 9 months, respectively. Postintervention, kapha was reduced $(-0.79)$ to within 0.29 of balance and further reduced at 9 months post $(-0.86)$ to within 0.22 of balance. Vata dosha was reduced $(-0.75)$ to within 0.14 of balance postintervention and pitta was reduced $(-0.29)$ to within 0.41 of balance postintervention.

\section{Other Ayurvedic outcomes}

Table 2 represents key Ayurvedic outcomes. The data show an additional $30 \mathrm{~min}$ of sleep per night on average, increasing from 7.06 to $7.58 \mathrm{~h}$, and a reduction in instances of waking by $50 \%(12-6)$ for study participants. Scales were developed to measure kapha-type moods such as depression, confusion, and feeling "stuck" or hurt with postintervention measures showing a reduction in frequency of kapha-negative moods of $48 \%$, as well as reduced severity of moods. Scales measuring kapha-negative cognitive patterns such as confusion, difficulty making decisions, and cloudy thinking showed a reduction of $57 \%$, with negligible increase in severity.

According to Ayurvedic theories of metabolism, adequate water intake is important for supporting metabolic activity and regular bowel movements promote detoxification. Both of these measures improved among study participants, who also reported a reduction in appetite at 3 and 6 month time points. All of these outcomes would be consistent with pacification of aggravated doshas and a hewing of doshic expression closer to the constitutional baseline.

Both Ayurveda and Yoga place a high emphasis on selfawareness and intentionality as keys to positive health and self-regulation. Study participants were given both standard (in yoga classes) and tailored tools for increasing selfawareness, positivity, and intentionality in their relationships with self and others. Ayurveda and Yoga consider these strategies to be critical to developing a positive affinity for behavior change and creating social support for new habits. A self-report instrument was designed to measure positive versus negative interactions on a weekly basis, intensity of interaction (either positive or negative), and whether they were intentional or involuntary.

Data in Table 2 show that postintervention, negative personal/ family interactions were down $7 \%$ and positive personal/ family interactions were up $10 \%$, the intensity of these interactions had increased by $11 \%$, and nonintentional interactions had decreased by $30 \%$. Positive work/school interactions increased $10 \%$ and decreased $3 \%$ with increased intensity of $20 \%$ and nonintentionality reduced by $9 \%$. Positive interactions with health and food increased $3 \%$ postintervention and negative interactions with health and food were reduced by $28 \%$, whereas intensity increased by $23 \%$ and involuntary 
interaction reduced by $6 \%$. Data were also collected on positive and negative interactions with self, showing that postintervention positive interactions with self were up by $7 \%$, negative interactions with self were reduced by $12 \%$, intensity was down $5 \%$, and nonintentional interaction was down $13 \%$. It is possible that these variables may be partially responsible for the improvements in psychosocial measures and lower perceived stress displayed in Table 1.

\section{Discussion}

This was the first known whole-systems Ayurvedic medicine and Yoga therapy intervention for obesity, including innovative instruments designed to collect Ayurvedic outcomes and incorporating dual diagnosis and dual outcomes collection from the perspectives of both Ayurveda and biomedicine. Data from the study demonstrate that the intervention was well tolerated by participants, with a satisfaction rate averaging $>90 \%$ at all time points. The study protocol included collection of both conventional and Ayurvedic outcomes, without presenting undue burden for participants.

Attendance at yoga classes averaged $75 \%$, combined yoga and home practice averaged $60 \%$, whereas adherence to dietary advice averaged 55\%. Thrice weekly yoga classes were well tolerated by individuals with a BMI up to 42 . Participants reported that the Ayurvedic outcome instruments promoted self-awareness around food and lifestyle choices. There was evidence of weight loss in $75 \%$ of participants, with percentage of weight loss increasing with combined yoga and dietary adherence.

Weight loss was correlated with adherence to yoga classes, home yoga practice, and compliance with dietary change suggestions. Participants reported overall benefits of the program two times as often as challenges associated with the program. ${ }^{18}$ Psychosocial outcomes and self-efficacy data contextualized the differential responses of completers versus dropouts and inform refinement of the program to address identified challenges to establishing and maintaining lifestyle change.

The semistandardized dietary modification encompasses a flexible eating restraint approach, de-emphasizing rigid dietary rules in favor of implementing self-care-oriented knowledge grounded in a commitment to a value-based goal. An Ayurvedic focus on experiential knowledge of food qualities and mindful healthy eating, rather than portions and calorie control, led participants to feel more competent and independent in maintaining protocol adherence and able to establish durable integration of lifestyle changes. Identified challenges included lack of family support, and a desire for assistance transitioning to community yoga classes during the follow-up period, to promote sustained practice over time. No adverse events associated with the dietary changes or yoga practices were reported.

\section{Limitations}

This study did not include a control group, randomization, or blinding. The unique study instruments developed to collect data on Ayurvedic medical features of the intervention will need to be validated in future studies. The small sample size of this pilot study means that this study was not powered to detect statistical significance for quantitative outcomes. Data collection and protocol adherence were estimated to be $65 \%$. Based on participant feedback, it is anticipated that retention and adherence rates will increase with refinement of the instruments and protocol, and flexibility in yoga class scheduling. On exit interview, participants cited the desirability for postintervention support groups to facilitate continued diet and lifestyle changes and booster yoga classes during follow-up ( $>3$ months) to promote home yoga practice. This integrated WSAY approach reflects a highly innovative clinical trial design, which may not be replicated in all Ayurvedic practices.

\section{Conclusions}

An Ayurveda-/yoga-based lifestyle modification program is an acceptable and feasible approach to weight management. Data collection, including self-monitoring and conventional and Ayurvedic outcomes, did not unduly burden participants, with attrition similar to that of other weight loss studies. Weight loss of $3.5 \mathrm{~kg}$ postintervention and $5.9 \mathrm{~kg}$ at 6 month follow-up shows proof of promise for a sustainable weight loss associated with well-integrated lifestyle changes that also increased self-reported vitality, psychosocial wellbeing, enhanced quality of life, and positive aspects of selfawareness and relationships. Integrated WSAY for obesity shows promise of durable health benefit and potential prevention or risk mitigation of other chronic conditions based on known comorbidities of obesity/obesity sequela.

\section{Acknowledgments}

At the time of the study, both authors were supported by the National Institutes of Health, Center for Integrative Medicine and Health T32 grant through the Department of Family and Community Medicine at the University of Arizona Medical School. The authors thank their mentor Cheryl Ritenbaugh, PhD, MPH, and Charis Domador, who selflessly served study participants as the yoga instructor and project assistant for this pilot study.

\section{Author Disclosure Statement}

No competing financial interests exist.

\section{References}

1. National Institutes of Health National Heart, Lung, and Blood Institute. Obesity, Nutrition and Physical Activity. 2016. Online document at: https://www.nhlbi.nih.gov/ science/obesity-nutrition-and-physical-activity Accessed November 8, 2018.

2. Hales CM, Carroll MD, Fryar CD, Ogden CL. Prevalence of Obesity Among Adults and Youth: United States, 20152016. Centers for Disease Control and Prevention. National Center for Health Statistics, NCHS Data Brief, No. 288, October 2017. U.S. Department of Health and Human Services, Washington DC, 2017.

3. Finkelstein EA, Trogdon JG, Cohen JW, Dietz W. Annual medical spending attributable to obesity: Payer- and service-specific estimates. Health Aff (Millwood) 2009;28: w822-w831.

4. US Department of Health and Human Services. The Surgeon General's call to action to prevent and decrease overweight and obesity. Online document at: www.surgeongeneral.gov/ 
library/calls/obesity/CalltoAction.pdf, accessed December 17, 2013.

5. Obesity and Overweight: Key Facts. World Health Organization, Geneva, Switzerland, 2018. Online document at: https://www.who.int/news-room/fact-sheets/detail/obesityand-overweight

6. Grover SA, Kaouache M, Rempel P, et al. Years of life lost and healthy life-years lost from diabetes and cardiovascular disease in overweight and obese people: A modelling study. Lancet Diabetes Endocrinol 2015;3:114-122.

7. Tsai AG, Williamson DF, Glick HA. Direct medical cost of overweight and obesity in the USA: A quantitative systematic review. Obes Rev 2011;12:50-61.

8. Ochner CN, Tsai AG, Kushner RF, Wadden TA. Treating obesity seriously: When recommendations for lifestyle change confront biological adaptations. Lancet Diabetes Endocrin 2015;3:232-234.

9. Miras AD, le Roux CW. Can medical therapy mimic the clinical efficacy of physiological effects of bariatric surgery? Int J Obes (Lond) 2014;38:325-333.

10. Knecht S, Ellger T, Levine JA. Obesity in neurobiology. Prog Neurobiol 2008;84:85-103.

11. Tate DF, Jeffery RW, Sherwood NE, Wing RR. Long-term weight losses associated with prescription of higher physical activity goals. Are higher levels of physical activity protective against weight regain? Am J Clin Nutr 2007;85: 954-959.

12. Rucker D, Padwal R, Li SK, et al. Long term pharmacotherapy for obesity and overweight: updated meta-analysis. BMJ 2007;335:1194-1199.

13. Li G, Zhang P, Wang J, et al. The long-term effect of lifestyle interventions to prevent diabetes in the China Da Qing Diabetes Prevention Study: a 20-year follow-up study. Lancet 2008;371:1783-1789.

14. Okura T, Nkata Y, Ohkawara K, et al. Effects of aerobic exercise on metabolic syndrome improvement in response to weight reduction. Obesity 2007;15:2478-2484.

15. Delgado-Floody P, Caamaño-Navarrete F, Gonzalez Rivera $\mathrm{J}$, et al. Improvements in Chilean patients with obesity following a 5-month multidisciplinary exercise program: a feasibility study. J Sports Med Phys Fitness 2018;58:309317.

16. Zolotarjova J, Ten Velde G, Vreugdenhil ACE. Effects of multidisciplinary interventions on weight loss and health outcomes in children and adolescents with morbid obesity. Obes Rev 2018;19:931-946.

17. Foster D, Sanchez-Collins S, Cheskin LJ. Multidisciplinary team-based obesity treatment in patients with diabetes: Current practices and the state of the science. Diabetes Spectr 2017;30:244-249.

18. Herman PM, Craig BM, Caspi O. Is complementary and alternative medicine (CAM) cost-effective? A systematic review. BMC Complement Altern Med 2005;5:11. Review.

19. Roux L, Kuntz KM, Donaldson C, Goldie SJ. Economic evaluation of multidisciplinary obesity intervention. Obesity (Silver Spring) 2006;14:1093-1106.

20. Igel LI, Saunders KH, Fins JJ. Why weight? An analytic review of obesity management, diabetes prevention, and cardiovascular risk reduction. Curr Atheroscler Rep 2018;20:39.

21. Sharma PV. Caraka Samhita, 9th ed. Chaukhambha Orientalia, India, 2005.

22. Kumar S, Shukla R, Sunil RB, Tiwari A. Non-pharmacological approach in the management of obesity (sthaulya). Sch J App Med Sci 2014;2:694-698.
23. Gaurav K, Sharma M, Kaundal R, et al. A review of sthaulya (obesity) and its management in ayurveda. UJAHM 2014;02: 66-72.

24. Rioux J, Howerter A, Thompson C. A pilot feasibility study of whole-systems ayurvedic medicine and yoga therapy for weight loss. Global Adv Health Med 2014;3:29-35.

25. Witt CM, Michalsen A, Roll S, et al. Comparative effectiveness of a complex Ayurvedic treatment and conventional standard care in osteoarthritis of the knee-Study protocol for a randomized controlled trial. Trials 2013;14: 149.

26. Kessler CS, Ostermann T, Meier L, et al. Additive complex ayurvedic treatment in patients with fibromyalgia syndrome compared to conventional standard care alone: A Nonrandomized Controlled Clinical Pilot Study (KAFA Trial). Evid Based Complement Alternat Med 2013;2013: 751403.

27. Patel MV, Patel KB, Gupta S, et al. A complex multiherbal regimen based on ayurveda medicine for the management of hepatic cirrhosis complicated by ascites: nonrandomized, uncontrolled, single group, open-label observational clinical study. Evid Based Complement Alternat Med 2015; 2015:613182.

28. Frawley D, Kozak SS. Yoga for your Type: An Ayurvedic Approach to Your Asana Practice. Twin Lakes, WI: Lotus Press, 2001.

29. Ayurvedic Yoga Therapist Professional Guidelines. National Ayurvedic Medical Association. Online document at: www .ayurvedanama.org/ayurvedic-yoga-therapist-programs, accessed January 3, 2019.

30. Lauche R, Langhorst J, Lee MS, et al. A systematic review and meta-analysis on the effects of yoga on weight-related outcomes. Prev Med 2016;87:213-232.

31. Rioux J, Ritenbaugh C. Narrative review of yogic interventions including weight-related outcomes. Altern Ther Health Med 2013;19:46-60.

32. Cramer H, Thoms MS, Anheyer D, et al. Yoga in women with abdominal obesity: A randomized controlled trial. Dtsch Arztebl Int 2016;113:645-652.

33. Yu AP, Ugwu FN, Tam BT, et al. One year of yoga training alters ghrelin axis in centrally obese adults with metabolic syndrome. Front Physiol 2018;9:1321.

34. Yang K, Bernardo LM, Sereika SM, et al. Utilization of 3 month yoga program for adults at high risk for type 2 diabetes: A pilot study. Evid Based Complement Alternat Med 2011;2011:257891.

35. Ross A, Brooks A, Touchton-Leonard K, Wallen G. A different weight loss experience: A qualitative study exploring the behavioral, physical, and psychosocial changes associated with yoga that promote weight loss. Evid Based Complement Alternat Med 2016;2016:2914745.

36. Ochner CN, Tsai AG, Kushner RF, Wadden TA. Treating obesity seriously: when recommendations for lifestyle change confront biological adaptations. Lancet Diabetes Endocrinol 2015;3:232-234.

37. Braun TD, Park CL, Gorin AA, et al. Group-based yogic weight loss with ayurveda-inspired components: A pilot investigation of female yoga practitioners and novices. Int $\mathbf{J}$ Yoga Therap 2016;26:55-72.

38. Lamba N. Review Article: The concept of medoroga \& its management through ayurveda. IAMJ 2017;5:156161.

39. Parauha S, Hullur MA, Prashanth AS. Sthaulya-A Life Style Disorder. SSRG-IJMS 2017;4:4-7. 
40. Tiwari N, Srivastava A, Manglesh R. An innovative approach for management of obesity through ayurveda: A review. Int J Res Ayurveda Pharm 2017;8:137-139.

41. Lad V. Textbook of Ayurveda, vol. 3: A Complete Guide to Clinical Assessment. Albuquerque, NM: Ayurvedic Press, 2006.

42. Rioux J. A complex, nonlinear dynamic systems perspective on Ayurveda and Ayurvedic research. J Altern Complement Med 2012;18:709-718.

43. Rioux JG. Yoga therapy research: A whole-systems perspective on comparative effectiveness and patient-centered outcomes. Int J Yoga Therap 2015;25:9-19.

44. Yank K, Rioux J. Yoga therapy for metabolic syndrome and weight control. In: Khalsa, SB, Cohen, L, McCall, T, Telles, S, eds. Principles and Practice of Yoga in Health Care. London, UK: Handspring Publishing, 2016.

45. Lad VD. Secrets of the Pulse: The Ancient Ayurvedic Art of Pulse Diagnosis, 2nd ed. Albuquerque, NM: Ayurvedic Press, 2006.

46. DuBroff R, Lad V, Murray-Krezan C. A Prospective trial of ayurveda for coronary heart disease: A pilot study. Altern Ther Health Med 2015;21:52-62.

47. de Toro-Martín J, Arsenault BJ, Després JP, Vohl MC. Precision nutrition: A review of personalized nutritional approaches for the prevention and management of metabolic syndrome. Nutrients 2017;9. pii: E913.

48. Dutton GR, Phillips JM, Kukkamalla M, et al. Pilot study evaluating the feasibility and initial outcomes of a primary care weight loss intervention with peer coaches. Diabetes Educ 2015;41:361-368.

49. Belfort-DeAguiar R, Seo D. Food cues and obesity: Overpowering hormones and energy balance regulation. Curr Obes Rep 2018;7:122-129.

50. Teixeira PJ, Carraça EV, Marques MM, et al. Successful behavior change in obesity interventions in adults: a systematic review of self-regulation mediators. BMC Med 2015;13:84.

51. Gould D, Kelly D, Goldstone L, et al. Visual Analogue Scale (VAS). J Clin Nurs 2001;10:697-706.

52. Flower A, Lewith G. A prospective case series exploring the role of Chinese herbal medicine in the treatment of recurrent urinary tract infections. Eur J Integr Med 2012;4: e421-e428.

53. Bandura A. Toward a psychology of human agency. Perspect Psychol Sci 2006;1:164-180.

54. Cohen S, Janicki-Deverts D. Who's stressed? Distributions of psychological stress in the United States in probability samples from 1983, 2006 and 2009. J Appl Soc Psychol 2012;42:1320-1334.

Address correspondence to: Jennifer Rioux, PhD, AD, AYT, IAYT Integral Ayurveda and Yoga Therapy 209 Lloyd Street, \#290 Carrboro, NC 27510

E-mail: integralayurvedayoga@gmail.com

\section{Appendices}

\section{Appendix A: Ayurvedic Dietary Guidelines for Weight Loss Study}

Emphasize lightly cooked or raw vegetables, whole grains and fresh fruits, and light proteins (legumes, fish, or poultry).

Please avoid sweet, salty, oily, heavy, rich, and dense foods. Gradually work toward eliminating processed and packaged foods, white flour and sugar, alcohol and dairy.

Drink 4-6, 8 oz glasses of room-temperature water per day. Drink one immediately upon waking and one glass with every meal. Please do not put ice in your drinks.

When eating, the stomach should be filled with $1 / 3$ food, $1 / 3$ water and $1 / 3$ space. Eat your biggest meal at midday.

Separate fruit from other foods by $30 \mathrm{~min}$ and do not eat meat and dairy together.

Please note any craving or avoidance of certain foods on your food wheel and your lifestyle change log.

Approach food intake as an act of self-love. Do not eat when feeling emotional or upset. Take a few minutes to focus on your breathing, perhaps reading the 5-element meditation. Consider your food as the source of your energy and vitality. Eat with awareness, focusing on the tastes and qualities of the foods you are eating, and how they make you feel.

Cook at least five meals at home per week and use the following spices: cumin, coriander, fennel, cardamom, ginger, cinnamon, turmeric, basil, oregano, mustard seeds, garlic, and black pepper.

\section{Appendix B: Yoga Sequence for Weight Loss Study (In-class and Home Practice)}

- In all poses, the light quality through suspension, distance from the ground, and lifting in limbs and trunk were emphasized. As sessions progressed, focus was on lengthening the duration of balance poses, increasing endurance in strength poses, more rapid transitions between poses, and maintaining longer pose sequences in a continuous flow. The slow, static, grounded, heavy, dense, and cool properties of kapha dosha, and increased oppositional qualities through speed of movement and raising heat in the body were de-emphasized.

- Beginning in week 5 of the study, sun salutations were added to the beginning of the sequence, as increased strength and endurance of participants made this practice accessible. The number of sun salutation repetitions was gradually increased over time to a maximum of four, preceding the start of the regular yoga sequence.

- Participants were instructed to practice Ujjayi pranayama throughout the yoga sequence and they were provided with a manual of all yoga poses, including photos.

\section{Mountain}

Mountain with arms overhead

Mountain with bound arms overhead 
Mountain with eagle pose arms

Mountain with cow face arms

Mountain with reverse namaste arms

Tree pose

Triangle

Warrior II

Extreme side angle

Half moon pose

Mountain

Chair

Straight-legged standing forward bend Plank

Upward dog

Down facing dog

Warrior I

Wide legged standing forward bend

Downward facing dog
Straight-legged standing forward bend Sitting on heels, toes bent under

Hero

Twisted hero

Seated twist

Spinal twist seated on floor

Upward facing head to knee

Wide legged seated forward bend

Cobbler pose

Camel pose

Reclining hand to foot pose

Bridge pose

Alligator twists

Legs up the wall (hips on blanket)

Corpse pose- $10 \mathrm{~min}$

5 Min SO/HUM meditation 